\title{
ALGUNAS CONCEPCIONES DE LOS ALUMNOS DE LA UNIVERSIDAD PEDAGÓGICA NACIONAL EN TORNO A LA PEDAGOGÍA Y LA DIDÁCTICA
}

\author{
Jorge Enrique Zuluaga Castro \\ Prof Cat. Depto. Química, Universidad Pedagógica Nacional
}

\begin{abstract}
This paper take the apreciations and observation of a group of students of the University Pedagógica Nacional of Colombia. That refers to the concepts that have with the pedagogic an didactic, their place of activity like the diferences.

They take of base information give of the students start of an aplica tions of cuestionary or enteview strutured that was realized during october of 2000.

This writing study 4 question of questionary to know: ¿That is the ocupation of pedagogic? ¿ What is the ocupation of didactic? ¿ What diference are in pedagogíc and didactic? and last ¿what forces and debilities indefies in the process of formation academic?
\end{abstract}

\section{RESUMEN}

Este escrito toma las apreciaciones y observaciones de un grupo de estudiantes de la Universidad Pedagógica Nacional de Colombia. Se refiere a los conceptos que se tienen sobre la pedagogía y la didáctica, así como las diferencias que ellos identifican.

La recopilación de información se hizo a partir de la aplicación de un instrumento durante el mes de octubre del año 2000.

Se estudian cuatro aspectos ¿De que se ocupa la pedagogía? ¿De qué se ocupa la didáctica? ¿Qué diferencias identifican entre pedagogía y didáctica? y por último se hace un análisis de las fortalezas y debilidades que identifican en su proceso de formación académica.

\section{INTRODUCCIÓN}

El artículo que a continuación se presenta, recoge las apreciaciones de un grupo de 21 alumnos de últimos semestres de diferentes carreras y facultades de la Universidad Pedagógica Nacionallos que en su mayoría, son estudiantes de la Facultad de Ciencia y Tecnología, de los Departamentos de Química, Diseño Tecnológico, Licenciatura en Electrónica, Física y Biología. En un menor número se recogen apreciaciones de alumnos de las Facultades de Educación Física, de la Facultad de Artes del Departamento de Música, así como de Psicología y Pedagogía y Licenciatura en Español Inglés, de la Facultad de Educación.

Las apreciaciones y observaciones de los alumnos están referidas a las concepciones que ellos tienen sobre la pedagogía, la didáctica y sus ámbitos de actividad y el 
posicionamiento que le atribuyen los alumnos en el contexto de las diferentes disciplinas y las ciencias.

Se buscó propiciar una reflexión crítica de estos campos de conocimiento, buscando identificar en ellos las apreciaciones, así como las posturas y opiniones respecto de las particularidades de estos campos de saber y conocimiento; su autonomía e interacciones, como las generalizaciones que desde la opinión de ellos se han venido construyendo y son dominios o espacios reclamados por la pedagogía y la didáctica, como de la factibilidad de elaboración de discursos autónomos y generales de estos saberes.

La construcción de discursos rigurosos y sistemáticos permite el posicionamiento de un saber o conocimiento, factor que además obliga a la contrastación de estas construcciones con otras teorías rivales y por ende a su validación o superación y el consecuente cambio de paradigmas.

Se consideró importante propiciar reflexiones individuales en estos grupos de estudiantes, las que en parte darán su contribución a autocontrastaciones sobre las concepciones que se tienen y manejan frente a estos tópicos, buscando que el entrevistado o encuestado(a) estableciera o atribuyera las diferencias entre lo pedagógico y didáctico, así como los ámbitos de actuación correspondientes de estos campos disciplinares.

El referido ejercicio reflexivo por parte de los estudiantes se realizó mediante la aplicación de un instrumento que se efectué indistintamente como en-cuesta o entrevista; esto, en razón de que la mayoría de preguntas se hacieron en forma abierta, permitiendo con ello que el entrevistado no quedara circunscrito solamente a las estrechas posibilidades que da una pregunta cerrada que solo ofrece unas posibilidades de respuesta limitada.

De esta manera, las indagaciones recogidas pueden ser entendidas como un "ejercicio etnográfico (si se permite la expresión) con ese particular grupo humano que son los estudiantes de la Universidad Pedagógica Nacional", aspirantes por demás a optar al título de Pedagogos y Didactas en una ciencia particular.

Si bien se indaga por la cultura pedagógica y didáctica que tienen los alumnos entrevistados, debe quedar claro desde el principio, que no indaga o averigua sobre todas las relaciones, sus componentes, como de los marcos conceptuales o enfoques, y corrientes pedagógicas y didácticas que circulan en el ámbito académico de la Universidad; Se indaga solo sobre aquellas concepciones, enfoques y relaciones que el (los) alumno(s) ha(n) venido elaborando y reelaborando respecto de sus marcos y estructuras conceptuales y representacionales.

En tal sentido debe entenderse, que no se busca establecer generalizaciones a partir de proposiciones particulares y menos a partir de una reducida muestra, sino mas bien de mostrar aspectos de los discursos particulares que circulan con alguna generalidad en la vida universitaria de los alumnos.

Otros aspectos que se indagan tienen que ver con elementos evaluativos y de autocrítica, que los alumnos se hacen sobre su proceso cumplido en la vida académica y de la formación que como activo intelectual han construido en su paso por la universidad; evaluación o autorreflexión que ellos hacen identificando las fortalezas y debilidades deducidas de su proceso de formación. 


\section{CONCEPCIONES DE LOS ESTUDIANTES FRENTE AL OBJETO DE LA PEDAGOGÍA}

La mayoría de los alumnos coinciden en señalar que la pedagogía se ocupa de la relación enseñanza-aprendizaje de un saber o conocimiento. De tal suerte la pedagogía se concibe como eje de preocupaciones o de problemas de estudio de dicha disciplina las relaciones e interacciones maestro-alumno. Esta perspectiva se ve plasmada en apreciaciones como la siguiente: "La pedagogía se ocupa de como el maestro y el alumno interactuan entre sí para buscar el método más eficaz en el proceso de enseñanzaaprendizaje.

Se ve en esta apreciación una clara orientación a concebir a pedagogía como unas formas, metodologías o estrategias, sin que en esta opinión se precise una diferenciación con la didáctica como se observará más adelante al revisar este aspecto. Lo anterior no quiere insinuar que la pedagogía como tal no deba a su interior establecer un cuerpo y estructura conceptual como unas reglas de producción del conocimiento o saber, que le es propio o pertinente, como lo es la educación y sus conceptualizaciones más próximas: las reflexiones sistemáticas sobre la relación enseñanza-aprendizaje y los métodos.

Un importante número de alumnos identifica en sus apreciaciones, que la pedagogía "se ocupa del quehacer educativo que es el conocimiento". Sin hacer mayores precisiones, frente la construcción y producción de conocimiento, queda en el tapete la pregunta, de si se asimila ella una epistemología, como si la pedagogía fuese una teoría del conocimiento, lo cual parece no ser objeto de ella como tal, sino, de espacios o ámbitos de trabajo de la filosofía, la epistemología o teorías del conocimiento, y en fin como ejercicio al que más debe dar respuesta, la filosofía de la ciencias, como campos de análisis de la producción de conocimiento o de saber de las ciencias y disciplinas particulares, sean ellas experimentales o no.

Otro sector de estudiantes en su indiferenciación conceptual en torno a la pedagogía y didáctica, manifiesta en sus opiniones, posiciones cercanas a lo que Commenio conceptualizará como didáctica, quien argumentaba que esta (la didáctica) es el arte de enseñar todo a todos en el menor tiempo posible. Allí podemos situar apreciaciones de los alumnos al responder "que ella (la pedagogía) busca el método más eficaz en el proceso de enseñanza-aprendizaje', o en aquella que sitúan a la "pedagogía como el arte de enseñar", o "la virtud o capacidad de enseñar", o "aquella que propone que ja pedagogía es la ciencia que hace fácil el proceso de evaluación". (Las frases entre comillas corresponden a las respuestas emitidas por los estudiantes).Es posible observar aquí, esa indiferenciación conceptual en donde la pedagogía se concibe como lo que Commenio entendia por didáctica.

Otros sectores de estudiantes se alejan de esa perspectiva meramente metodológica e instrumental, queriendo darle un sustrato de reflexión teórica y conceptual, incluyendo en dicha perspectiva la contextualización histórica política y social de la actividad educativa y por ende de la enseñanza y el aprendizaje.

Veamos algunos ejemplos de esta intencionalidad: "La pedagogía se ocupa de la reflexión teórica sobre ¡a praxis educativa... en este sentido debe valerse de las ciencias de la educación tales como la psicología de la educación... Consecuentemente se ocupa de la teorización acerca de la formación de hombres sociales para su proyecto de 
sociedad específica". (Las frases entre comillas corresponden a las respuestas emitidas por los estudiantes).

En forma análoga posturas como, "que la pedagogía se ocupa de realizar a través de un conocimiento (disciplina) la enseñanza de este con un enfoque que pretende la conscientización de este conocimiento al trasladarlo al contexto social en el queso desenvuelve el estudiante...". "En otras palabras es adaptar ese conocimiento no como factor enajenado de su realidad sino como factor presente en todas las actividades que se realizan a diario. También se ocupa del análisis y crítica de todos los actos y situaciones que hubo, hay o habrá en el sector educativo, político, económico, social, cultural, etc. Y buscar los posibles aportes que se puedan adoptar para el mejoramiento de las condiciones sociales, en que se encuentra el medio".

Otras posturas que presentan paralelismo a estas últimas, considera que "la pedagogía busca compilar todas las formas y métodos de enseñanza y aprendizaje que se han desarrollado a través de la historia del hombre y determinar cuales son las más apropiadas para ser aplicadas a nivel educativo, en busca de brindar una educación pertinente a la época y circunstancias".

Otro tanto ocurre con aquellos que consideraron que "la pedagogía se ocupa de los procesos de educación enmarcados dentro de una comunidad. Como tal considera el hombre como ser individual y lo que trata de potenciar es su máximo desarrollo para servicio del entorno social".

Se dibujan en estas posiciones al menos dos aspectos que se consideran pertinentes de señalar, en aras de la precisión de los ámbitos del discurso y quehacer pedagógico.

De una parte queda en las apreciaciones referidas, la sensación de que la pedagogía debe apoyar el proceso de incorporación del individuo a la estructura social y cultural a la cual pertenece y de otra parte la asignación de un papel y responsabilidad de la disciplina pedagógica, para direccionar mediante o a través de la educación el proyecto de hombre y sociedad que se pretende acercar y/o alcanzar.

En esta óptica vale puntualizar la existencia de una acriticidad sobre el modelo social, cultural, político y económico en el que se inscribe el educando, al asignarle a la pedagogía un papel importante sino fundamental en la reproducción del modelo socio cultural en tal sentido se puede caer en un repeticionismo mecanisista de los sabores, sin abordar una crítica de su producción y finalidad, es decir la formación y socialización del individuo, sin buscar el desarrollo de una reflexión critica del modelo social y cultural en el cual se inscribe y del que forma parte el estudiante.

De otra parte se percibe una intencionalidad y teleología social para la pedagogía cuando de ella se espera que produzca sobre la base del reconocimiento y comprensión del modelo social una crítica de la sociedad misma como de los problemas objeto de estudio de la pedagogía y su papel frente a la sociedad. Así las cosas la pedagogía se acerca a lo que podemos concebir como ideología y las finalidades políticas que se puedan derivar. Con estas apreciaciones se sitúa a la pedagogía no en un campo propio de la reflexión teórico conceptual de la educación, y la relación enseñanza y aprendizaje sino como una ideologización.

Se busca asignarle a la pedagogía un papel clave en el proceso de reproducción social, todo ello debido al papel que juega en el proceso de formación de los individuos y 
grupos humanos. La población y sociedad se educa no solo en la incorporación de elementos de la cultura material o plataforma instrumental y tecnológica, sino que además integra a su proceso de incorporación social la complejidad de los sistemas de organización social, sistemas de conocimiento y sistemas simbólicos y religiosos.

Si la pedagogía se debe ocupar de definir el proyecto de sociedad y de ser humano que se pretende construir y de desarrollar los mecanismos y estrategias educativas para la incorporación de estos al modelo y dinámicas sociales, nos adentramos en otro campo de reflexión que no corresponde rigurosamente a la pedagogía.

Si la pedagogía se ocupa de "un sistema de ideas creencias y actitudes normativas, y supuestamente basadas en hechos mantenidos colectivamente se define un modo particular de relaciones y acuerdos sociales y/o tendente a justificar un modelo de conducta, que sus defensores intentan promover, procurar o mantener" (Dobson, 1997). Estamos hablando no de pedagogía sino de ideología en el sentido de como la entiende M. Hamilton.

El enfoque que se propone a continuación guarda relación con lo anterior en el sentido de asignarle a la pedagogía una función de teleología social.

Hay opiniones que dan a la pedagogía un papel fundamentalmente teórico en el campo del proceso de interacción enseñanza - aprendizaje "teniendo en cuenta las particularidades del individuo, el contexto histórico, geográfico y socio económico; los avances científicos y tecnológicos. Además de ser una elaboración teórica es dialéctica, cambiante y relacionada con el objeto que la sociedad pretende alcanzar con sus instituciones educativas y la educación en general".

De otro lado se aprecian enfoques y posturas que ven "la pedagogía como el arte o la virtud de enseñar". Pero ha de ser claro que el problema de la educación tampoco se resuelve teniendo tales condiciones artísticas o de virtuosismo. Si estimamos que la educación involucra otros problemas (transformación de jas propias concepciones, comprensión de procesos, observación, reflexión, análisis y evaluación, entre otros) y que ella no se resuelve solo con tener un buen maestro aunque ello sea de gran importancia en el proceso. El campo de construcción teórica se amplia y se abre a nuevas preguntas.

Cuando se propone que ja pedagogía se ocupa de la transmisión del conocimiento y de las formas expeditas para que el maestro los comunique, el estudiante está imbuido de una actitud pasiva frente al conocimiento y el saber como si el maestro tuviese encriptado el conocimiento y él y solo él fuera el "dador de saber y conocimiento". En tal concepción el alumno es un sujeto pasivo, el maestro sabe, comunica y el estudiante se ilumina con el saber recibido, como si fuese una actividad mecánica. Pero claro ese proceso está mediado por un complejo movimiento intelectual: conflictos, contrastaciones con las concepciones que tenía el sujeto, comprensión de nuevas relaciones, como reflexiones sobre las nuevas implicaciones de tales interacciones.

Por último otra concepción reflejada en las opiniones de los estudiantes, ubica a "la pedagogía como la ciencia de la educación en la que se debe llegar a una profunda reflexión educativa en la que se deben discutir, las directrices de la educación, comenzando por el aula y siguiendo por la instrucción para culminar en la socialización ante la comunidad educativa general". Esta apreciación amarra la construcción de pedagogía al ámbito de su actividad: el sector educativo institucionalizado y a sus comunidades académicas. En esta perspectiva en ja medida que se configuren estas y sus 
espacios de investigación como programas de la comunidad académica se entrará en terrenos de cada vez mayor sistematización y rigurosidad, y de selectividad y posicionamiento de nuevos paradigmas para la pedagogía y la educación.

\section{DE LAS CONCEPCIONES DE LOS ALUMNOS SOBRE DIDÁCTICA}

En este campo, en el proceso de indagación efectuado, se observan como dominantes las posturas que entienden la didáctica, ya como una disciplina auxiliar de apoyo de ja pedagogía, o como una actividad eminentemente metodológica e instrumental: "la didáctica es una herramienta de la pedagogía". "Es la parte de la pedagogía que se ocupa de la enseñanza "“Es la que facilita los medios para llegar a una buena pedagogía

"En donde esta presta contribuciones a aquella para el cumplimiento de sus objetivos, la incorporación el conocimiento por el sujeto que aprende".

Otra orientación que se observó corresponde al grupo de alumnos que la entienden como "la disciplina que se ocupa de los mecanismos apropiados para una buena transmisión de conocimientos", o la conciben como metodología, forma, estrategia o herramienta y su aplicación para facilitar o hacer más eficiente y eficaz el aprendizaje del alumnado.

Estas opiniones se sitúan en el terreno instrumental al concebirse la didáctica como métodos, estrategias y herramientas. Esta orientación supone dar a la didáctica un perfil positivista en la medida que las herramientas y métodos didácticos permiten llevar a análogas transformaciones en los alumnos con lo que se convalidarían permanentemente los métodos, estrategias e instrumentos utilizados en el proceso educativo, a esta mecánica positiva se circunscribirían los procesos educativos que quedarían resueltos desde esos métodos infalibles. No obstante la evidencia de que en el fondo subyace una gran complejidad y diversidad en los sujetos cognocentes hace pensar que no unas únicas o ya definidas metodologías de enseñanza agotan y resuelven el asunto.

Métodos de enseñanza, para la comprensión y aprendizaje son entre otros, elementos que en forma interactuante hacen parte del proceso educativo, de transformación intelectual, sin embargo concepciones que están impregnadas del convencimiento de que los conocimientos se transmiten y se adoptan sin más, están a la orden del día en las concepciones que manejan los alumnos.

Se piensa como si el aprendizaje fuera solo asunto de los maestros que lo proporcionan y facilitan con sus métodos y no como también evidentemente ocurre, de los alumnos y de sus autotransformaciones conceptuales, epistemológicas, y de actitud, al entrar en contrastación sus ordenamientos conceptuales, creencias actitudes, con lo que se propone por el maestro, o con lo que en su momento se constituya como objeto de estudio, frente a sus concepciones ( ya sea naturaleza de la sociedad o de constitución de la materia, la luz, la vida, la naturaleza, la energía, la realidad, lo cotidiano, lo artificial, etc., según sea el objeto de estudio o problemas en estudio)

El proceso de conocer en muchos casos implica crisis en la medida que constituye una lucha entre las viejas concepciones (las que ha elaborado tiene el alumno), con las que presentan los artificios rigurosos de construcción de la razón humana, hechos teorías y formulaciones desde las ciencias frente a las concepciones y comportamiento de los 
fenómenos (sean la naturaleza de las sociedades o de la constitución de la materia, la luz, la vida, etc.)

\section{DIFERENCIACIONES PUNTUALES ENTRE PEDAGOGÍA Y DIDÁCTICA DESDE LA PERCEPCIÓN DE ALGUNOS ALUMNOS DE LA UNIVERSIDAD PEDAGÓGICA NACIONAL}

Aunque hay una tendencia a reconocer la pedagogía como ja teoría y la didáctica como las herramientas para ¡levar a la práctica lo educativo, se delinean otras aproximaciones y variaciones al respecto. Una diferenciación conceptual de los términos pedagogía y didáctica, desde las representaciones de jos alumnos entrevistados se aluden a continuación.

Hay quienes consideran que la pedagogía se ocupa de la formación de la persona, ya en su condición de ser social e histórico y la didáctica se ocuparía de la formación académica disciplinaria. Con esto se está orientando la postura a que la pedagogía se debe ocupar de la discusión ética y política del sujeto y su sociedad y que la didáctica tiene su tareas en la formulación de métodos estrategias e instrumentos expeditos para ja transmisión o comunicación de conocimientos, elaborados y validados en la sociedad.

Se observan también posturas contrarias que más que una nueva versión teórica, denota la confusión reinante, estas se manifiestan en discursos circulares que no apuntan a dar claridad sobre el aspecto y solo dejan ver definiciones insinuadas con artificiosos juegos de palabras que no pasan de ser malabarismos lingüísticos sin nuevas implicaciones semánticas y de construcción de conocimiento. Se hace referencia a afirmaciones como "la pedagogía es un método de enseñanza y la didáctica un recurso de esta" o "la didáctica es un instrumento pedagógico y la pedagogía la ciencia que la utiliza".

De nuevo se manifiesta la concepción instrumental de la actividad didáctica como aquella que se encarga de ingeniar artificios para facilitar el aprendizaje.

Otro sector de alumnos opté por no responder o por explicitar su propia confusión frente a la diferenciación conceptual de la pedagogía y la didáctica "mis límites se confunden". "No tengo claridad ni elementos para precisar las diferencias". En tal sentido, una cuarta parte de los informantes se manifestó de esta forma.

Se resalta que las concepciones se localizan entre aquellos que piensan que la pedagogía se refiere en forma genérica a teorías y corrientes para la transmisión del conocimiento y aquellas que refieren la didáctica a los métodos de transmisión, o su versión emparentada en donde la pedagogía es la teoría de la educación y la didáctica jas formas, estrategias y métodos de enseñanza.

\section{QUE FORTALEZAS Y DEBILIDADES IDENTIFICAN LOS ESTUDIANTES EN SU PROCESO DE FORMACIÓN}

Mientras que algunos identifican unos aspectos como fortalezas en otras esas mismas se constituyen en debilidades.

Veamos: Hay quienes ven como una fortaleza en su proceso deformación, la construcción de una mayor autoestima, autoconfianza, que conlleva a unos mejores niveles de 
autovaloración. Otros identifican en la diversidad de actores una falta de identidad y compromiso llamando la atención sobre los aspectos actitudinales no solo de los alumnos sino y también de los profesores. "Mis maestros ya están cansados de enseñar, les falta empeño, ganas de hacer jas cosas bien" de sí mismos algunos reconocen sus vacíos al explicitar, "hay falta de rigurosidad en mis lecturas acerca de estos temas".

Muchos luego de haber cursado más de la mitad de la carrera consideran que sólo hasta ahora están incursionando en el saber pedagógico sintiendo fortalezas en ja formación disciplinar especifica.

Cerca del $60 \%$ de los informantes opina que su formación pedagógica y didáctica es deficiente frente a los retos que en un futuro próximo tendrá que asumir como educador.

El hecho de que los jóvenes en ocasiones no tengan argumentos para presentar sus posturas, habla no solo de sus deficiencias en la formación disciplinar específica como también de las deficiencias discursivas y lingüísticas de sus actitudes (aspectos afectivos y conductuales) frente al conocer.

Del campo disciplinar específico (química, biología, geografía, música, etc) como de otra especificidad como lo es la formación pedagógica y didáctica, condición requerida para ja constitución de comunidades académicas y de investigación, en estos campos y para el avance de estos, además de ser el objeto y razón de ser de sus estudios.

Al revisar las apreciaciones de jos informantes al respecto de la importancia que ellos indican, se le ha conferido al componente pedagógico, se podrían encontrar razones que explican los vacíos anotados en su formación pedagógica y didáctica.

Es común encontrar opiniones de los alumnos, que consideran que hay un gran vacío en los currículos de los diferentes programas académicos, considerando que la formación pedagógica y didáctica está relegada a un segundo plano, aunque se resalta la importancia de tener una buena formación en estos campos dado el perfil profesional para el cual se están formando.

En forma análoga las apreciaciones de los alumnos frente a la importancia que ellos estiman se le ha conferido a la formación didáctica, indican tanto la importancia de la formación en este campo, como de las deficiencias que ellos mismos ven en su formación didáctica.

Al respecto veamos algunas apreciaciones de los alumnos: "Se diría que la formación didáctica es media-baja, el currículo jatiene relegada a un segundo plano. "Es muy importante la formación en este campo, pero aunque se hace énfasis en su utilización, no se profundiza mucho en sus métodos y es muy poco aplicada por los maestros en nuestra enseñanza". "En la universidad he oído hablar mil veces del tema, pero siento que no hay claridad. "Son pocas las veces que puedo señalar donde se nos ha guiado como futuros docentes, sobre las posibles maneras de llevar una clase." Si acaso se que quiere decir didáctica".

Estas apreciaciones no son muy consideradas frente a los esfuerzos que realizan los profesores, ya que algunos alumnos son de la opinión de que en los docentes hay falta de creatividad y que las actividades propuestas por los maestros son repetitivas y monótonas. 
Pareciera mostrarse en la actitud de los alumnos, su pasividad frente a su proceso de formación didáctica, la que se mimetiza tras la oportunidad de presentar sus observaciones críticas al respecto. Se busca justificar y responsabilizar de ese vacío al currículo o a los profesores como si su proceso de formación tanto pedagógica didáctica y disciplinar específica, no fuera también y fundamentalmente responsabilidad de si mismo, es decir del alumno. Se acoge aquí la apreciación de que nadie aprende por otro (Gallego B. 1999)

Por lo demás este aspecto referido hace evocar a las personas moralmente dependientes que se estudian y describen en la teoría de la psicología de la cognición de Jean Piaget y Laurence Koelberg (Cadutto M. 1994). Se hace evidente con las apreciaciones de los alumnos los bajos niveles de compromiso y de responsabilidad frente a su futuro papel como educadores.

Esta referencia a lo actitudinal frente a ja reflexión pedagógica salta a la vista con la falta de participación e interés por estructurar unos discursos, lo que se vio reflejado en la baja participación de informantes tanto del sector de estudiantes como de profesores vale aclarar para el lector que de 308 formularios entregados que buscaban indagar sobre enfoques y aspectos referidos a lo pedagógico y didáctico luego de un mes solo se conté con las apreciaciones de 29 personas entre profesores (8) y estudiantes (21), la revisión de las apreciaciones hechas por los profesores que informaron en el cuestionario, serán motivo de otro escrito.

\section{BIBLIOGRAFIA}

Ávila, Rafael. ¿Qué es la pedagogía? Bogotá: Editorial Nueva América, 1990.

Bachelard, Gaston. El racionalismo aplicado. Buenos Aires: Paidós, $1^{\text {a }}$ de 1978.

Wolches Chaux, Gustavo. La letra con risa entra. Fundación FES, Ecofondo, Fondo FEN Colombia. Popayán, 1996.

Briones, Guillermo. Metodología de la investigación en Ciencias Sociales.

Dobson, Andrew. Pensamiento político verde. Editorial Paidós, 1997.

Cadutto, Michel. Guía metodológica para la formación de valores ambientales.

Gallego Badillo, Rómulo. El saber pedagógico. Bogotá: Editorial Magisterio, 1992.

El trabajo pedagógico. Bogotá: Universidad Pedagógica Nacional, 1996.

Gallego B. Rómulo; Pérez Miranda, Royman. El problema del cambio en las concepciones epistemológicas y didácticas. Bogotá: Editorial Universidad Pedagógica Nacional, 1999.

Competencias cognocitivas. Bogo-Editorial Magisterio, 1999.

Gallego Badillo, Rómulo y Gallego Torres, Adriana Patricia. Reflexiones metodológicas en torno a la pedagogía. Inédito Bogotá, 2000.

Popper, Karl. La lógica de la investigación científica. Madrid: Editorial Tecnos, 1962. 
Tames García, Maria Adela; Forero, Aura; Garzón, Teresa; Nieto, María Emma; Parra, Olga Lucía; Rodríguez, Ana C. Historia de la pedagogía.

Zubiria, Miguel De. Tratado de pedagogía conceptual. Bogotá: Fundación Alberto Merani, 1994. 